\title{
The quest for a universal antidote
}

Antidotes provide a direct way to counteract acute adverse effects of a drug; for example, protamine can be used to combat bleeding due to administration of the anticoagulant heparin. However, the rational design of antidotes is challenging and costly, so they are not available for most approved therapeutics. Reporting in Nature Medicine, Sullenger and colleagues describe antidote molecules for a whole class of drugs - those based on aptamers.

Aptamers are small singlestranded nucleic-acid molecules that bind and inhibit protein targets. So far, one aptamer drug that binds to vascular endothelial growth factor has been approved for age-related macular degeneration. However, aptamers are also being developed as anticoagulants and for conditions such as cancer and HIV.

Previous studies with anticoagulant aptamers have shown that customized oligonucleotide sequences that base pair with aptamers to form duplex RNAs prevent aptamer activity. However, this is not a viable approach to generate antidotes for many clinical applications as developing such customized oligonucleotides is prohibitively expensive. In addition, the resulting aptamer-antidote complex could trigger an immune response, which might exacerbate the adverse effect of the aptamer.

The authors observed that two anticoagulant aptamers that had different primary sequences, secondary structures and targets in the coagulation cascade (factor IXa and factor Xa) could both be inactivated by protamine. This prompted them to further examine the possibility that a single antidote might bind to and counteract the activity of several aptamers. They carried out a screen of DNA or small interfering RNA delivery polymers to identify other nucleic-acid-binding molecules that provide antidotes to these two aptamers with more favourable properties than protamine, which is associated with adverse effects such as a sudden fall in blood pressure.

Several polymers were found to completely reverse the effects of these aptamers, and their potency as antidotes correlated with their affinity for the aptamers. Two of these polymers $-\beta$-cyclodextrincontaining polycation (CDP) and polyphosphoramidate polymer (PPA-DPA) - were able to rapidly neutralize the anticoagulant effects of various aptamers in whole blood, suggesting that they are sequenceindependent antidotes. Furthermore, the authors showed that sequential administration of an anticoagulant aptamer and CDP led to the formation of an aptamer-polymer complex in mice. In pigs, CDP rapidly and durably (within 5 minutes and for at least 60 minutes) reversed the effects of the aptamer on activated clotting time without any signs of toxicity.

Although the safety of this type of agent will have to be further tested in clinical studies, the possibility of designing antidotes to aptamers, regardless of their sequence and structure, could subtantially enhance the safety profile of aptamer-based therapeutics and might encourage their development for a wider range of indications.

Monica Hoyos Flight

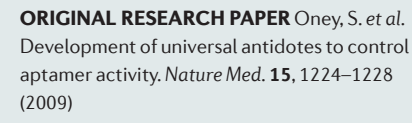

ORIGINAL RESEARCH PAPER Oney, S. et al. Development of universal antidotes to control aptamer activity. Nature Med. 15, 1224-1228 (2009)

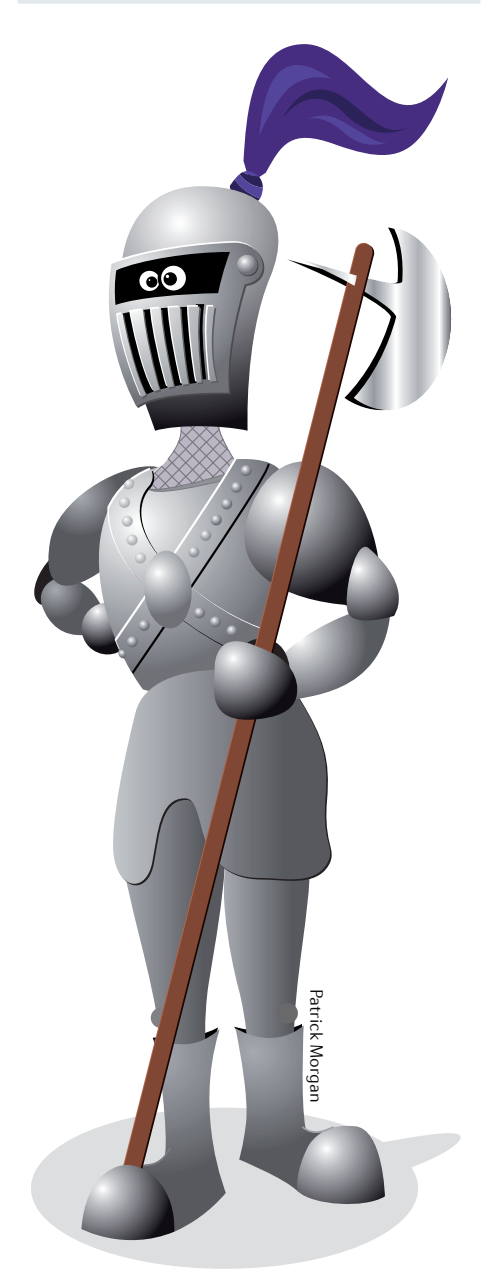

